\title{
Multiple Annular Keloidal Plaques: A Clinical Case
}

\author{
Mason Henehan, Paula Beers, Vladimir Vincek and Anna De Benedetto* \\ Department of Dermatology, University of Florida College of Medicine, Gainesville, FL, USA
}

\begin{abstract}
Granuloma annulare (GA) and keloids are distinct entities with classic clinical and pathologic findings, but the pathogenesis of each has yet to be fully elucidated. GA is proposed to be due to a cell-mediated, delayed-type hypersensitivity reaction while keloids are hypothesized to be driven by TGF- $\beta$. This case report describes a 56 -year-old African-American female with multiple annular and polycyclic plaques on the trunk which demonstrate histopathology of GA and keloid at different locations. Such an unusual presentation suggests the possibility of GA inciting a keloidal response, a phenomenon that has been sparsely documented in the literature to date.
\end{abstract}

Keywords

Granuloma annulare, GA, Keloid, Scarring

\section{Introduction}

Granuloma Annulare (GA) is a non-life threatening, granulomatous dermatosis of uncertain pathogenesis with a preference for females and mean onset after 50 years of age. Clinically, it typically appears as non-scaly, skin-colored or erythematous papules coalescing into an annular plaque. Lesions are most commonly localized in an acral distribution but can be generalized [1]. Histologically, classical GA is characterized by lymphohistiocytic infiltrates, mucin deposition, and either an interstitial or palisading pattern of histiocytes with necrobiotic collagen forming granulomas [2]. The underlying mechanism of GA has been postulated to be a cell-mediated, delayed-type hypersensitivity reaction to an unknown antigen with secondary vessel changes rather than a primary vasculitis [3]. Cytokines IFN- $\gamma$ and TNF- $\alpha$ have been implicated in the disease process [4]. Another hypothesis builds on the finding that GA patients have impaired neutrophil chemotaxis in vivo, which may predispose them to macrophage-dominant granulomatous reactions [5]. Nonetheless, mild findings of microvascular damage provide a theoretical basis for the debated association between diabetes mellitus and GA. This association has been especially well documented for generalized GA $[1,6]$. A recent review by Piette and Rosenbach outlines numerous conditions and triggers possibly associated with GA, including diabetes mellitus, dyslipidemia, autoimmune thyroiditis, and HIV [7].

Keloids are benign proliferations of dense fibrous tissue, classically characterized by aberrant scarring that extends beyond the borders of the original wound [8]. They are more common in darker skin types [9] and tend to appear in puberty and pregnancy [10]. A slight predominance of females over males has been noted, but this may be due to higher rates of earlobe piercing [11]. Clinically, lesions typically begin as pink to erythematous nodules or plaques with symptoms of pain and/or pruritus, and usually do not spontaneously regress [8]. There is a predilection for the chest, ears, shoulders, head, and neck regions [12]. Histologically, keloids have irregularly oriented whorls of thickened, hyalinized collagen, as well as other features such as a "tongue-like advancing edge" beneath the epidermis [13]. The pathogenesis of keloids is believed to involve increased sensitivity of fibroblasts to TGF- $\beta$ causing excess collagen production [14].

GA and keloids are distinct entities with sparse evidence of linkage in the literature. We present a case with an unusual pattern of annular keloidal plaques with histopathology demonstrating a coexistence of these two conditions, suggesting the possibility that GA could result in keloid development.

\section{Case Presentation}

A 56-year-old African-American female presented to our outpatient clinic with multiple pruritic and tender lesions on her trunk. The patient reported that for several years she had had extensive keloid scarring over the chest, abdomen, and back, and that these lesions were painful and itchy. The first lesion had developed at the site of her Cesarean section 21 years prior, with development of adjacent lesions thereafter. Some also arose in response to bug bites and minor skin

*Corresponding author: Anna De Benedetto, MD, Department of Dermatology, College of Medicine, University of Florida, 4037 NW $86^{\text {th }}$ Terrace, $4^{\text {th }}$ Floor, Gainesville, FL 32606, USA

Accepted: November 19, 2018

Published online: November 21, 2018

Citation: Henehan M, Beers P, Vincek V, et al. (2018) Multiple Annular Keloidal Plaques: A Clinical Case. Dermatol Arch 2(1):68-71 


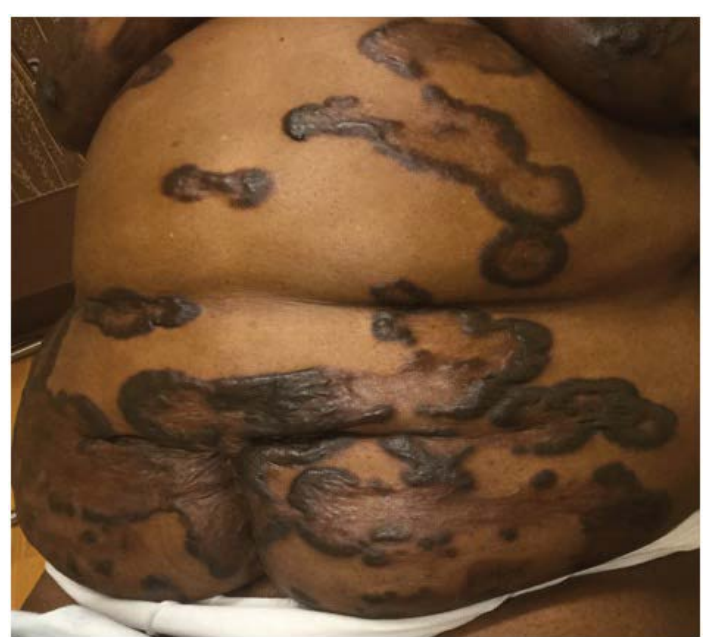

A

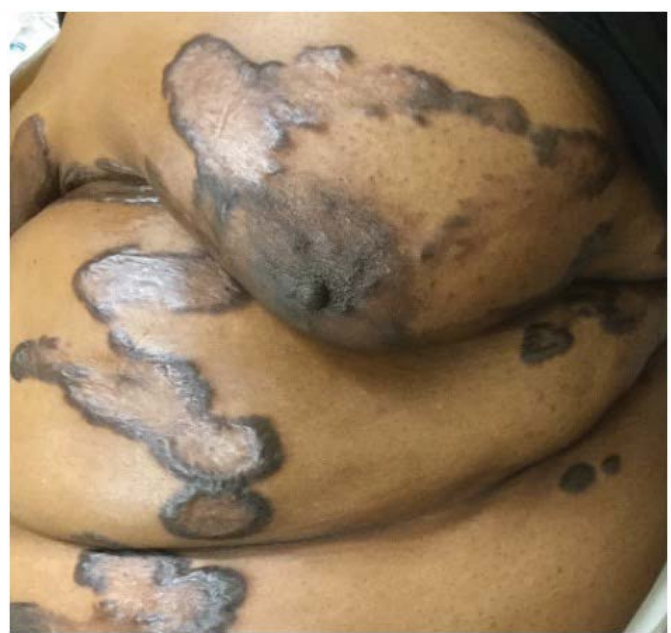

B

Figure 1: A,B) Extensive annular and polycyclic plaques with nodular borders on the abdomen and breasts.

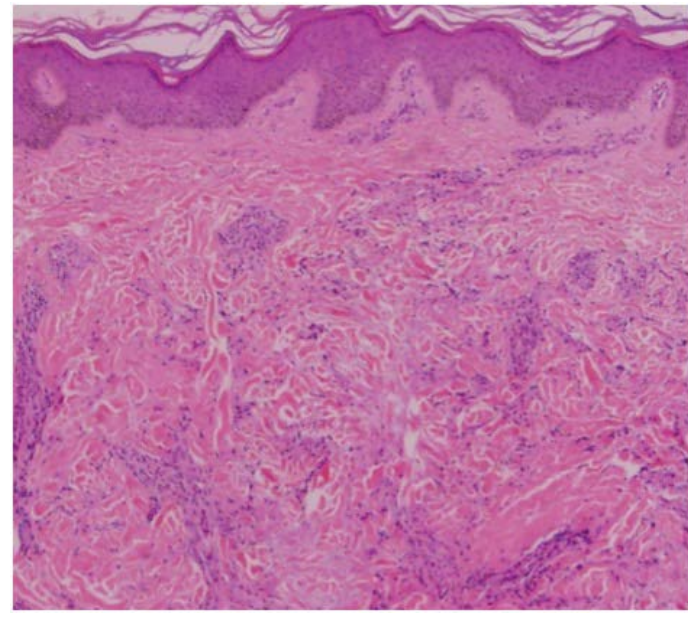

A

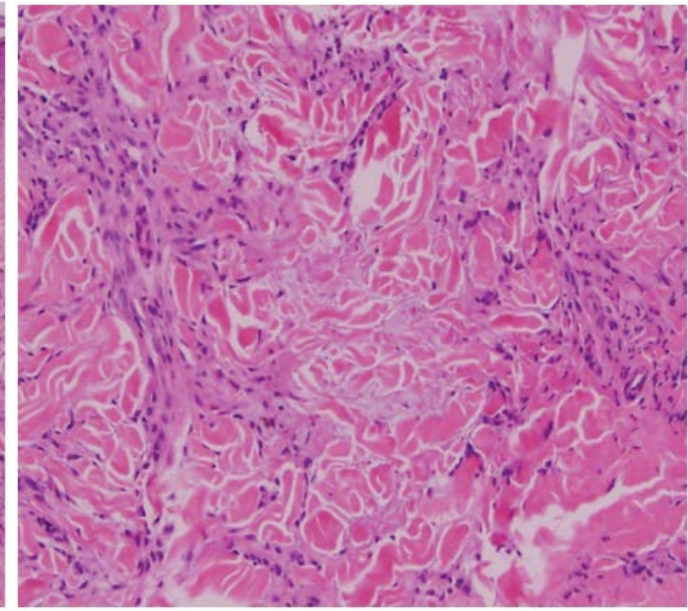

B

Figure 2: A) Lymphocytic and histiocytic infiltrate surrounding necrobiotic collagen characteristic of GA, 40x; B) Lymphocytic and histiocytic infiltrate with necrobiosis, 100x.

trauma, but others appeared without apparent cause. Several lesions had progressed initially, but all lesions had been stable over the past few years. Her primary concern was the intense pruritus, for which she was using only topical Vaseline without much relief. The patient reported having problems wearing underwear or tight-fitting clothing because of a "pain" on the skin related to these lesions. Previously, she had visited other dermatology offices for the same problem and was repeatedly given a diagnosis of keloids. She had had steroid injection of keloids on the ear in the past as well but did not appreciate much improvement after two treatments and was deterred by the pain associated with the procedure. Her past medical history consisted of only diabetes and hypertension and was otherwise unremarkable. She had no other known skin conditions. She was up-to-date with her age-appropriate cancer screening. She denied personal or family history of autoimmune disease or sarcoidosis. As per her social history, the patient admitted to smoking, but denied drinking alcohol or using illicit drugs.
Physical exam revealed multiple annular and polycyclic smooth plaques with nodular, hyperpigmented, fibrotic borders and central clearing with an absence of scale or other secondary changes (Figure $1 \mathrm{~A}$ and Figure $1 \mathrm{~B}$ ). Despite the pruritic nature of the plaques, they were not lichenified. There was no warmth or rim of erythema present to suggest active irritation of the plaques. Lesions were distributed across the chest, abdomen, and back in a random pattern; the face, legs, mucous membranes, and palmoplantar skin were spared. No calcinosis cutis, sclerodactyly, or Raynaud phenomenon were observed on the hands, and the nails appeared normal. Two punch biopsies were performed from representative lesions: one from left lateral abdomen and the other from left medial abdomen. One biopsy showed a normal epidermis with lymphocytic and histiocytic inflammation surrounding foci of necrobiotic collagen in an interstitial pattern in the superficial dermis consistent with granuloma annulare (Figure 2A and Figure 2B). The other biopsy demonstrated a somewhat thickened epidermis with scant chronic inflammation and 


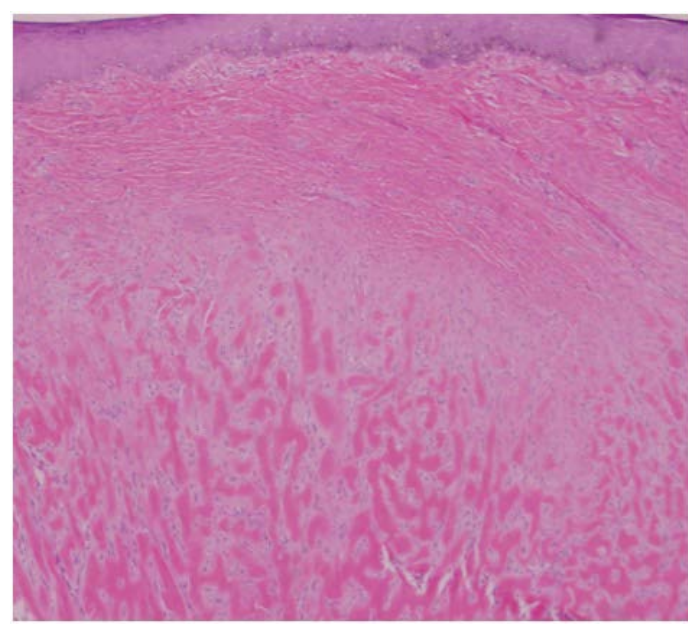

A

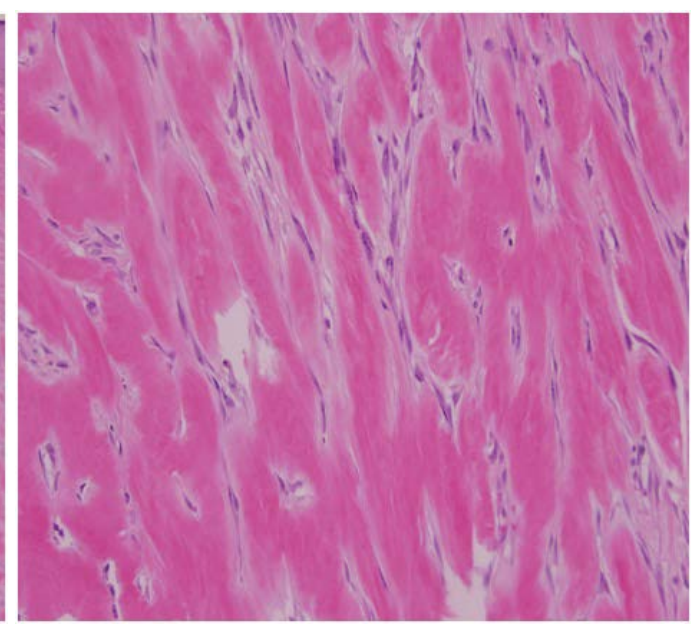

B

Figure 3: A) Eosinophilic hyalinized keloidal collagen bundles, 40x; B) Keloidal collagen bundles with surrounding fibroblasts, 100x.

large bundles of fibroblasts surrounding thick eosinophilic collagen bundles typical of a keloid (Figure $3 \mathrm{~A}$ and Figure 3B). An Alcian blue stain was performed on the specimen with features of GA, which was positive for the presence of mucin. A partially ruptured hair follicle with intrafollicular and perifollicular acute inflammation was also observed.

The patient was initially treated with topical triamcinolone ointment for symptomatic relief of pruritus (pending biopsy results) but had minimal effect on reducing itch. After the pathology was reviewed, she was treated with a brief course of doxycycline for folliculitis and was planned to start a trial of hydroxychloroquine for treatment of generalized GA but was lost to follow-up.

\section{Discussion}

We present a case of extensive keloids with annular and polycyclic morphology on the trunk of a middle-aged AfricanAmerican woman with unique histopathologic features at different sites. Initially, the clinical differential diagnosis included keloids, GA, annular sarcoidosis, and nodular scleroderma. Given the unusual presentation of the keloids as annular plaques with central clearing along with the pathology findings, we suggest the possibility of generalized GA contributing to keloid formation. In the literature, there are only rare anecdotal considerations of an association between GA and keloids. One review of 47 cases from 1967 found 3 patients with both entities [15]. An early GA case series from 1908 discusses 3 patients with lesions that clinically appeared as GA but histologically were determined to be keloid; the author hypothesized that there is a "keloid-like stage" in the involution of GA [16]. A more recent case in the literature described GA arising years after a keloid scar, in which the keloid was secondary to a lightning strike while wearing a gold chain [17]. In our patient, we are hesitant to label her histologically keloidal lesions as a "stage of involution" of GA since they have been stable in size and character on her skin for many years.

GA has also been described in some cases to arise as a Wolf isotopic response to a herpes zoster scar. Interestingly, all 5 patients in a case series of GA isotopic response showed peri neurovascular and/or perifollicular inflammation - similar to our patient in which one biopsy showed perifollicular inflammation [18]. Thus, an alternative hypothesis in our case would be that the folliculitis could be a contributor to one or both of these aberrant responses. However, given how atypical it would be for keloids or folliculitis to form multiple polycyclic configurations on their own, we favor GA as the initial insult to the skin in this patient.

A non-classical presentation of keloids should also prompt clinicians to initially consider nodular scleroderma, a rare variant of scleroderma that presents with keloidal lesions as opposed to classic sclerotic plaques. Case reports have described this condition both as presenting in patients with systemic sclerosis with variable histopathologic findings, as well as in individuals with no systemic involvement but with histologic evidence of morphea $[19,20]$. In our case, symptoms of scleroderma were absent, and biopsy was also not consistent, although a more thorough workup was unable to be completed.

In conclusion, the interest of this case rests on the concurrent presence of keloids and GA. This is intriguing from an immunologic perspective, as keloids are believed to be driven by TGF- $\beta$ [14], while one study of GA lesions found no detectable TGF- $\beta$ by Northern hybridization (suggesting collagen production in GA is driven by a different pathway) [21]. Since these two conditions are not frequently encountered together and seem to have stark differences in pathophysiology, this patient should prompt further scientific investigation into these two important dermatologic diseases.

\section{Acknowledgements}

None.

\section{References}

1. Dabski K, Winkelmann RK (1989) Generalized granuloma annulare: Clinical and laboratory findings in 100 patients. J Am Acad Dermatol 20: 39-47.

2. Dabski K, Winkelmann RK (1989) Generalized granuloma 
annulare: Histopathology and immunopathology. Systematic review of 100 cases and comparison with localized granuloma annulare. J Am Acad Dermatol 20: 28-39.

3. Umbert P, Belcher RW, Winkelmann RK (1976) Lymphokines (MIF) in the serum of patients with sarcoidosis and cutaneous granuloma annulare. Br J Dermatol 95: 481-485.

4. Fayyazi A, Schweyer S, Eichmeyer B, et al. (2000) Expression of IFNgamma, coexpression of TNFalpha and matrix metalloproteinases and apoptosis of $\mathrm{T}$ lymphocytes and macrophages in granuloma annulare. Arch Dermatol Res 292: 384-390.

5. Gange RW, Black MM, Carrington P (1979) Defective neutrophi migration in granuloma annulare, necrobiosis lipoidica, and sarcoidosis. Arch Dermatol 115: 32-35.

6. Studer EM, Calza AM, Saurat JH (1996) Precipitating factors and associated diseases in 84 patients with granuloma annulare: a retrospective study. Dermatology 193: 364-368.

7. Piette EW, Rosenbach M (2016) Granuloma annulare: Pathogenesis, disease associations and triggers, and therapeutic options. J Am Acad Dermatol 75: 467-479.

8. Robles DT, Berg D (2007) Abnormal wound healing: keloids. Clin Dermatol 25: 26-32.

9. Brissett $A E$, Sherris DA (2001) Scar contractures, hypertrophic scars, and keloids. Facial Plast Surg 17: 263-272.

10. Koonin AJ (1964) The aetiology of keloids: A review of the literature and a new hypothesis. S Afr Med J 38: 913-916.

11. Kelly AP (2004) Medical and surgical therapies for keloids. Dermatol Ther 17: 212-218.
12. Bayat A, Arscott G, Ollier WE, et al. (2004) Description of sitespecific morphology of keloid phenotypes in an Afrocaribbean population. Br J Plast Surg 57: 122-133.

13. Lee JY, Yang CC, Chao SC, et al. (2004) Histopathological differential diagnosis of keloid and hypertrophic scar. Am J Dermatopathol 26: 379-384.

14. Bettinger DA, Yager DR, Diegelmann RF, et al. (1996) The effect of TGF-beta on keloid fibroblast proliferation and collagen synthesis. Plast Reconstr Surg 98: 827-833.

15. Stankler L, Leslie G (1967) Generalized granuloma annulare. A report of a case and review of the literature. Arch Dermatol 95: 509-513.

16. Little EG (1908) Granuloma annulare. Proc R Soc Med 1: 95-163.

17. Angit C, Clayton TH (2009) Lightning-strike granuloma. Clin Exp Dermatol 34: e485-e486.

18. Kapoor R, Piris A, Saavedra AP, et al. (2013) Wolf isotopic response manifesting as postherpetic granuloma annulare: a case series. Arch Pathol Lab Med 137: 255-258.

19. Rencic A, Brinster N, Nousari CH (2003) Keloid morphea and nodular scleroderma: two distinct clinical variants of scleroderma? J Cutan Med Surg 7: 20-24.

20. Kassira S, Jaleel T, Pavlidakey P, et al. (2015) Keloidal scleroderma: Case report and review. Case Rep Dermatol Med.

21. Kallioinen M, Sandberg M, Kinnunen T, et al. (1992) Collagen synthesis in granuloma annulare. J Invest Dermatol 98: 463-468.

DOI: $10.36959 / 661 / 302$

Copyright: (c) 2018 Henehan M, et al. This is an open-access article distributed under the terms of the Creative Commons Attribution License, which permits unrestricted use, distribution, and reproduction in any medium, provided the original author and source are credited. 